\title{
ON THE PARAMETERS OF LEWIS METRIC FOR THE WEYL CLASS
}

\author{
M. F. A. da Silva \\ Departamento de Física e Química, Universidade Estadual Paulista, \\ Av. Ariberto Pereira da Cunha 333, 12500, Guaratinguetá - SP, Brazil and \\ Departamento de Astrofísica, CNPq-Observatório Nacional, \\ Rua General José Cristino 77, 20921-400, Rio de Janeiro - RJ, Brazil. \\ L. Herrera \\ Departamento de Física, Facultad de Ciencias, \\ Universidade Central de Venezuela and \\ Centro de Física, Instituto Venezolano de Investigaciones Científicas, \\ Caracas, Venezuela. Postal address: Apartado 80793, Caracas 1080A, Venezuela \\ F. M. Paiva and N. O. Santos \\ Departamento de Astrofísica, CNPq-Observatório Nacional, \\ Rua General José Cristino 77, 20921-400, Rio de Janeiro - RJ, Brazil
}

\begin{abstract}
Author's internet addresses respectively: mfas@on.br, lherrera@conicit.ve, fmpaiva@on.br and nos@on.br.
\end{abstract}




\begin{abstract}
We provide physical interpretation for the four parameters of the stationary Lewis metric restricted to the Weyl class. Matching this spacetime to a completely anisotropic, rigidly rotating, fluid cilinder, we obtain from the junction conditions that one of these parameters is proportional to the vorticity of the source. From the Newtonian approximation a second parameter is found to be proportional to the energy per unit of length. The remaining two parameters may be associated to a gravitational analog of the Aharanov-Bohm effect. We prove, using the Cartan scalars, that the Weyl class metric and static Levi-Civita metric are locally equivalent, i.e., indistinguishable in terms of its curvature.
\end{abstract}




\section{Introduction}

Lewis stationary vacuum metric [1] is usually presented with four parameters [2] without being given a specific physical interpretation unless matched to a particular source. Here we shall provide physical interpretation for the four parameters characterizing the Lewis metric, when the source consists of a general rigidly rotating anisotropic fluid cylinder.

The Lewis metric solution of Einstein's equations can be split into two families called Weyl class and Lewis class. Here we shall restrict our study to the Weyl class where all four parameters appearing in the metric are real. For the Lewis class these parameters can be complex.

Matching the Weyl class metric to a cylindrical fluid, from the junction conditions one of the parameters is found to be proportional to the vorticity of the source. Examination of the Newtonian limit shows that a second parameter is proportional to the energy per unit length, also in the same limit a third parameter appears as the arbitrary constant potential which is always present in the Newtonian solution. Finally the fourth parameter, together with the first, is found to be responsible for the non-staticity of Lewis metric and has to vanish when the vorticity of the source tends to zero if one wishes to recover the Levi-Civita static vacuum metric [3], in that limit. Additionally these two last parameters may be related to a gravitational analog of the Aharanov-Bohm effect.

The physical properties of solutions of the Einstein's equations are not obvious from a given metric tensor, in part due to the coordinate arbitrariness in general relativity. Using an approach borrowed from the equivalence problem [4,5], and based on the Cartan scalars, we shall study the local properties of the Lewis spacetime. It is demonstrated that the Weyl 
class metric is indistinguishable of the Levi-Civita metric from the curvature point of view. However we show that they are topologically different.

The paper is organized as follows: in the next three sections we give the notation and the spacetime at both sides of the boundary of the source. In section 5 we introduce the Cartan scalars technic and apply it to investigate the local properties of the Lewis metric. In section 6 the junctions conditions are discussed. The Newtonian approximation is presented in section 7, together with a discussion about the gravitational Aharanov-Bohm effect. In section 8 we present a conclusion. Three appendixes are added, at the end, for the Ricci and Riemann tensor components and the algebraic invariants of the Riemann tensor. These quantities are calculated for the metric that we shall study.

\section{Spacetime}

The spacetime we describe by the general cylindrically symmetric stationary metric

$$
d s^{2}=-f d t^{2}+2 k d t d \varphi+e^{\mu}\left(d r^{2}+d z^{2}\right)+l d \varphi^{2}
$$

where $f, k, \mu$ and $l$ are functions only of $r$, and the ranges of the coordinates $t, z$ and $\varphi$ are

$$
-\infty<t<\infty, \quad-\infty<z<\infty, \quad 0 \leq \varphi \leq 2 \pi
$$

the hypersurfaces $\varphi=0$ and $\varphi=2 \pi$ being identified. The coordinates are numbered

$$
x^{0}=t, \quad x^{1}=r, \quad x^{2}=z, \quad x^{3}=\varphi .
$$

The metric (2.1) has to satisfy Einstein's field equations,

$$
R_{\mu \nu}=\kappa\left(T_{\mu \nu}-\frac{1}{2} g_{\mu \nu} T\right)
$$


given in its usual notation. The non zero components of the Ricci tensor $R_{\mu \nu}$ for (2.1) are given in the appendix A.

The spacetime is devided into two regions: the interior, with $0 \leq r \leq R$, to a cylindrical $\Sigma$ surface of radius $R$ centered along $z$; and the exterior, with $R \leq r<\infty$.

\section{Exterior spacetime}

The exterior spacetime is filled with vacuum, hence Einstein's equations (2.4) reduce to

$$
R_{\mu \nu}=0 .
$$

The general solution of (3.1) for (2.1) is the stationary Lewis metric [1] given in reference [2] notation,

$$
\begin{gathered}
f=a r^{-n+1}-\frac{c^{2}}{n^{2} a} r^{n+1}, \\
k=-A f, \\
e^{\mu}=r^{\frac{1}{2}\left(n^{2}-1\right)}, \\
l=\frac{r^{2}}{f}-A^{2} f,
\end{gathered}
$$

with

$$
A=\frac{c r^{n+1}}{n a f}+b
$$

The constants $a, b, c$ and $n$ can be either real or complex, the corresponding solutions belong to the Weyl class or Lewis class, respectively. Here we restrict our study to the Weyl class.

The transformation $[7]$

$$
d \tau=\sqrt{a}(d t+b d \varphi)
$$




$$
d \bar{\varphi}=\frac{1}{n}[-c d t+(n-b c) d \varphi]
$$

casts the metric (2.1) into

$$
d s^{2}=-r^{-n+1} d \tau^{2}+r^{\frac{1}{2}\left(n^{2}-1\right)}\left(d r^{2}+d z^{2}\right)+\frac{1}{a} r^{n+1} d \bar{\varphi}^{2} .
$$

This is locally the Levi-Civita metric. Nevertheless, since $\varphi=0$ and $\varphi=2 \pi$ are identified, $\tau$ defined in (3.7) attains a periodic nature unless $b=0[7]$. On the other hand, the new coordinate $\bar{\varphi}$ ranges now from $-\infty$ to $\infty$. A more detailed accounting on this subject can be found in Stachel $[8,9]$. In order to globally transform the Weyl class of the Lewis metric into the static Levi-Civita metric we have to make $b=0$ and $c=0$. Note that in this case, from the transformations (3.7) and (3.8), $\tau$ and $\bar{\varphi}$ become respectively true time and angular coordinates.

Hence we can say that $b$ and $c$ are responsible for the non-staticity of this family of solutions in the Lewis metric.

\section{Interior spacetime}

The interior spacetime is described by a cylinder filled with stationary anisotropic fluid, hence the energy momentum tensor $T_{\mu \nu}$ is given by

$$
T_{\mu \nu}=\left(\varrho+P_{r}\right) u_{\mu} u_{\nu}+P_{r} g_{\mu \nu}+\left(P_{\varphi}-P_{r}\right) K_{\mu} K_{\nu}+\left(P_{z}-P_{r}\right) S_{\mu} S_{\nu}
$$

where $\varrho$ is the energy density, $P_{r}, P_{z}$ and $P_{\varphi}$ are the principal stresses, and $u_{\mu}, K_{\mu}$ and $S_{\mu}$ are four vectors satisfying

$$
u_{\mu} u^{\mu}=-1, \quad K_{\mu} K^{\mu}=S_{\mu} S^{\mu}=1,
$$




$$
K_{\mu} u^{\mu}=S_{\mu} u^{\mu}=K_{\mu} S^{\mu}=0
$$

Considering the source under rigid rotation and choosing the coordinates in a comoving frame, we obtain for $u^{\mu}, K^{\mu}$ and $S^{\mu}$ using $(2.1)$ and $(4.2-3)$

$$
\begin{gathered}
u^{\mu}=\left(\frac{1}{\sqrt{f}}, 0,0,0\right), \\
K^{\mu}=\left(\frac{k}{D \sqrt{f}}, 0,0, \frac{\sqrt{f}}{D}\right), \\
S^{\mu}=\left(0,0, e^{-\frac{\mu}{2}}, 0\right) .
\end{gathered}
$$

Calculating the energy momentum tensor (4.1) using (2.1) and (4.4-6) we obtain that the non null components are

$$
T_{0}^{0}=-\varrho, \quad T_{1}^{1}=P_{r}, \quad T_{2}^{2}=P_{z}, \quad T_{3}^{3}=P_{\varphi}, \quad T_{3}^{0}=\frac{k}{f}\left(\varrho+P_{\varphi}\right)
$$

We observe that $T_{0}^{3}=0$, hence the corresponding component of Einstein's field equations (2.4) becomes

$$
\left(\frac{f k^{\prime}-k f^{\prime}}{D}\right)^{\prime}=0
$$

where we used (A.2). Integrating (4.8) we obtain

$$
f k^{\prime}-k f^{\prime}=\alpha D
$$

where $\alpha$ is an integration constant.

The vorticity tensor $\omega_{\alpha \beta}$ of the matter inside the cylinder is given by

$$
\omega_{\alpha \beta}=\frac{1}{2}\left(u_{\alpha ; \beta}-u_{\beta ; \alpha}\right)+\frac{1}{2}\left(u_{\alpha ; \nu} u^{\nu} u_{\beta}-u_{\beta ; \nu} u^{\nu} u_{\alpha}\right) .
$$

With (2.1) and (4.4) we obtain the non null components of (4.10)

$$
\omega_{31}=\frac{1}{2}\left(\frac{k^{\prime}}{\sqrt{f}}-\frac{k f^{\prime}}{f \sqrt{f}}\right)
$$


The vorticity vector $W^{\alpha}$ is

$$
W^{\alpha}=\frac{1}{2} \frac{\varepsilon^{\alpha \beta \gamma \delta}}{\sqrt{-g}} u_{\beta} \omega_{\gamma \delta}
$$

and with (2.1), (4.4) and (4.11) we obtain

$$
W^{\alpha}=\frac{1}{2} \varepsilon^{3 \alpha 10} \frac{1}{D e^{\mu}}\left(k^{\prime}-k \frac{f^{\prime}}{f}\right) .
$$

Now calculating the magnitude of $W^{\alpha}$ from (4.13) and considering (4.8) we have

$$
\left(W_{\alpha} W^{\alpha}\right)^{\frac{1}{2}}=\frac{\xi}{2 f e^{\frac{\mu}{2}}} .
$$

Hence we see from (4.14) that $\xi$ measures the vorticity of the cylinder.

Althought we did not solve all the interior field equations for the general source (4.1) it can be easily shown that solutions exist. In fact Krasiński $[10,11,12]$ found a complete set of solutions of the field equations for a perfect fluid source and matched his solutions to the corresponding exterior solutions [13]. Another and well known interior solution is that due van Stockum [14], with vanishing pressure. In this particular case the expression for the vorticity (4.14) reduces to the correspondent Bonnor result [7] where his $a$ is equal $-\frac{\xi}{2}$.

\section{Cartan scalars}

In this section, the equivalence problem and its solution due to Cartan [4] and Karlhede [5] are presented. We show how it leads to the Cartan scalars and to a local characterization of spacetimes. At the end of this section we apply this approach to the Weyl class metric $(3.2-5)$.

The equivalence problem consists of deciding whether a coordinate transformation $\tilde{x}^{a}=$ $\tilde{x}^{a}(x)$ exists which takes a metric $g_{a b}(x)$ into another metric $\tilde{g}_{c d}(\tilde{x})$. If it exists, these two 
metrics are called equivalent. A first attempt to solve this problem would probably be to use the scalars made by contraction of the Riemann tensor (the so called 14 algebraic invariants) and its covariant derivatives. Unfortunately, this fails, i.e., two metrics may have the same scalars (built in the above way) and be not equivalent. For example, these scalars are all zero for plane-waves and Minkowski spacetimes and yet they are not equivalent metrics [15]. We present in appendix $\mathrm{C}$ the non null 14 algebraic invariants of the Riemann tensor calculated for the metric (3.2-5) if the reader wants to compare them with the Cartan scalars obtained below. The best known solution to the equivalence problem was presented by Cartan [4] and can be summarized as follows.

Let $\omega^{A}(A=0, \ldots, 3)$ be a frame such that the line element can be written as $d s^{2}=$ $\eta_{A B} \omega^{A} \omega^{B}$. The components of the Riemann tensor and its covariant derivatives (up to possibly the $10^{\text {th }}$ order) calculated in a constant frame $\left(\eta_{A B}\right.$ constant) shall be called Cartan scalars. This name comes from the fact that the Cartan scalars transform like scalars under coordinate transformations. Notice, however, that they transform like tensor components under frame transformations. With this in mind, we can state the equivalence theorem, due to Cartan [4] as:

Theorem: Two metrics are equivalent if and only if there exist coordinate and Lorentz transformations which transform the Cartan scalars of one of the metrics into the Cartan scalars of the other.

As introduced above, the Cartan scalars are defined up to Lorentz and coordinate transformations. Lorentz transformations present no great problem since the frame may be uniquely fixed (up to isotropies of the metric) by choosing a standard form to the set of 
Cartan scalars. Once a standard frame is chosen, the Cartan scalars become defined up to coordinate transformations. Although there is no algorithm for deciding whether there is a coordinate transformation which takes one set of Cartan scalars into the other, it is, in most practical cases, a decidable task.

The practical algorithm for calculating the Cartan scalars was developed by Karlhede [5]. Here we present a summary (for recent reviews see, for instance [5,16,17]). Such an algorithm could have, in principle, 11 steps, from the $0^{\text {th }}$ order derivative up to the $10^{\text {th }}$. This number can be thought of as related to the 6 Lorentz transformations, the 4 coordinates of the spacetime and one integrability condition. At each order of derivation, the frame is fixed up to the isotropies of the Cartan scalars calculated until this order. At each order, the number of the functionally independent functions of the coordinates among this set of Cartan scalars is also found. How to fix the frame and how to find the independent functions are explained in, for instance, $[16,18]$. There will be a moment when no new information for fixing the frame and no new functionally independent function of the coordinates are found. At this point, no new derivatives are necessary [4] and the set of Cartan scalars is complete, in the sense that they are necessary and sufficient for deciding the equivalence problem.

Since the Cartan scalars are sufficient for deciding about the equivalence of metrics, they contain all local information about a metric and provide a local characterization of a spacetime. Nevertheless, global properties, such as topological deffects, would probably not appear in the Cartan scalars.

The Cartan scalars can be calculated by using the computer algebra system SHEEP [19], the program CLASSI [20] and their interface with REDUCE [15]. The practical implementation of the algorithm works with spinors rather than tensors, since symmetries are better handled in this way. MacCallum and Åman [21] found a minimal set of algebraically 
independent Cartan scalars in the spinorial formalism which, for vacuum solutions, as is the case of the Weyl class metric, are, until the first order:

$$
\begin{gathered}
0^{\text {th }} \text { derivative : } \Psi_{A}, A=(0, \ldots, 4) ; \\
1^{\text {st }} \text { derivative }: \nabla \Psi_{A B^{\prime}}, A=(0, \ldots, 5), B=(0,1) ;
\end{gathered}
$$

where $\Psi_{A}$ is the Weyl spinor and $\nabla \Psi_{A B^{\prime}}$ is the first symmetrized covariant derivatives of $\Psi_{A}$ [15].

In order to calculate the Cartan scalars for the Weyl class metric (3.2-5), we chose the following Lorentz tetrad:

$$
\begin{aligned}
\theta^{0} & =\sqrt{f} d t-\frac{k}{\sqrt{f}} d \varphi, \\
\theta^{1} & =\frac{1}{\sqrt{f}} r d \varphi, \\
\theta^{2} & =e^{\frac{\mu}{2}} d r, \\
\theta^{3} & =e^{\frac{\mu}{2}} d z .
\end{aligned}
$$

Since the Cartan scalars are calculated in the spinorial formalism, a null frame must be used, which we define from the above by

$$
\begin{aligned}
\omega^{0} & =\frac{1}{\sqrt{2}}\left(\theta^{0}+\theta^{1}\right), \\
\omega^{1} & =\frac{1}{\sqrt{2}}\left(\theta^{0}-\theta^{1}\right), \\
\omega^{2} & =\frac{1}{\sqrt{2}}\left(\theta^{2}+i \theta^{3}\right), \\
\omega^{3} & =\frac{1}{\sqrt{2}}\left(\theta^{2}-i \theta^{3}\right) .
\end{aligned}
$$


Following the usual technics [5], we find that the standard null frame is given by the transformation

$$
\begin{aligned}
\tilde{\omega}^{0} & =\left(\frac{n a-c r^{n}}{n a+c r^{n}}\right)^{\frac{1}{2}} \omega^{0}, \\
\tilde{\omega}^{1} & =\left(\frac{n a+c r^{n}}{n a-c r^{n}}\right)^{\frac{1}{2}} \omega^{1}, \\
\tilde{\omega}^{2} & =\omega^{2} \\
\tilde{\omega}^{3} & =\omega^{3} .
\end{aligned}
$$

The correspondent standard Lorentz frame can be found from this one using (5.2), which gives:

$$
\begin{aligned}
& \tilde{\theta}^{0}=n a\left(\frac{f}{n^{2} a^{2}-c^{2} r^{2 n}}\right)^{\frac{1}{2}}(d t+b d \varphi), \\
& \tilde{\theta}^{1}=\left(\frac{f}{n^{2} a^{2}-c^{2} r^{2 n}}\right)^{\frac{1}{2}} r^{n}[-c d t+(n-b c) d \varphi], \\
& \tilde{\theta}^{2}=e^{\frac{\mu}{2}} d r, \\
& \tilde{\theta}^{3}=e^{\frac{\mu}{2}} d z .
\end{aligned}
$$

In the standard null frame, the Cartan scalars are found to be

$$
\begin{gathered}
\Psi_{2}=-\frac{1}{8}\left(n^{2}-1\right) r^{-\frac{1}{2}\left(n^{2}+3\right)} \\
\Psi_{0}=\Psi_{4}=-n \Psi_{2} \\
\nabla \Psi_{01^{\prime}}=\nabla \Psi_{50^{\prime}}=-\frac{\sqrt{2}}{16} n\left(n^{4}-1\right) r^{-\frac{3}{4}\left(n^{2}+3\right)} \\
\nabla \Psi_{10^{\prime}}=\nabla \Psi_{41^{\prime}}=-\frac{\sqrt{2}}{8} n\left(n^{2}-1\right) r^{-\frac{3}{4}\left(n^{2}+3\right)} \\
\nabla \Psi_{21^{\prime}}=\nabla \Psi_{30^{\prime}}=\frac{\sqrt{2}}{32}\left(n^{2}+3\right)\left(n^{2}-1\right) r^{-\frac{3}{4}\left(n^{2}+3\right)}
\end{gathered}
$$

Since no new information appears in the first derivative, it is not necessary to find the second derivative. From the Cartan scalars, we can find that the metric is Petrov type I and 
therefore has no isotropy group and that it has a 3 dimensional isometry group, since the Cartan scalars present a single functionally independent function of the coordinates.

From the Cartan scalar we see that only the parameter $n$ helps to curve the spacetime for the Weyl class metric. Hence, it can be showed that the Cartan scalars for the LeviCivita metric (3.9) are identical to those of the Weyl class of the Lewis metric. Therefore, from the curvature point of view, Levi-Civita static metric (3.9) and the Weyl class stationary metric are indistinguishable, which confirms the coordinate analysis of the end of the section 3. However, as it will be shown below, these two metrics posses very different topological behaviour.

\section{Junction conditions}

On the surface of discontinuity $\Sigma, r=R$, the junction conditions are those of Darmois [22], namely, that the first and second fundamental forms have to be continuous. Choosing the same coordinates for the exterior and interior spacetimes these conditions become

$$
\begin{aligned}
& {\left[g_{\mu \nu}^{-}-g_{\mu \nu}^{+}\right]_{\Sigma}=0,} \\
& {\left[g_{\mu \nu}^{-\prime}-g_{\mu \nu}^{+\prime}\right]_{\Sigma}=0,}
\end{aligned}
$$

where the indexes - and + stand for the interior and exterior spacetimes respectively.

At $r=R$ considering (3.2-6), (4.9) and (6.1-2) we obtain

$$
c=-\frac{\xi}{2} .
$$


Hence, from (6.3) and (4.14) we have that the constant $c$ in the Weyl class metric measures the vorticity of the source described by a rigidly rotating anisotropic fluid cylinder. For the particular case of the null pressure Bonnor's result [7] is recovered.

\section{Newton limit and Ahanarov-Bohm effect}

In the Newtonian limit, the vorticity term is negligible, then from $(6.3) c \approx 0$, and calling $(3.2)$

$$
f=e^{2 U}
$$

then $U$ is

$$
U=2 \sigma \ln r+\frac{1}{2} \ln a
$$

where $\sigma$ is given by

$$
\sigma=\frac{1}{4}(1-n) .
$$

In Newton's theory, (7.2) is the gravitational potential of an infinite uniform line-mass with mass per unit length $\sigma$. The constant $\frac{1}{2} \ln a$ in (7.2) represents the constant arbitrary potential that exists in the Newtonian solution. Timelike circular geodesics exists for $1>n>0$, or

$0<\sigma<\frac{1}{4}$, as expected in the Newtonian analog. When $\sigma=\frac{1}{4}$ the circular geodesics become null $[6,23]$.

The metric (3.2-6) has infinite curvature, according to the Cartan scalars (5.5-8), only at $r=0$ for all $n$ except $n= \pm 1$, i.e., $\sigma=0$ and $\frac{1}{2}$ according to (7.3). Thus the Weyl class metric has a singularity along the axis $r=0$, then we can say that this spacetime is generated by an infinite uniform line source for densities $0<\sigma<\frac{1}{4}$. 
Considering the static limit for the Weyl class metric when $n=1(\sigma=0)$ and $b=c=0$ we have from $(2.1)$

$$
d s^{2}=-d t^{2}+d r^{2}+d z^{2}+\frac{r^{2}}{a} d \varphi^{2}
$$

giving rise to a locally flat spacetime. In this case (7.4) represents the spacetime generated by a string along the axis of symmetry with linear energy density $\lambda$ given by $[25]$

$$
\lambda=\frac{1}{4}\left(1-\frac{1}{\sqrt{a}}\right)
$$

being $a>1$. Hence the constant $a$ is directly linked to the gravitational analog of AharanovBohm effect [26]. This effect shows that gravitation depends on the topological structure of spacetime giving rise to an angular defficit $\delta$ equal to

$$
\delta=2 \pi\left(1-\frac{1}{\sqrt{a}}\right)
$$

as in the eletromagnetic Aharanov-Bohm effect, where a (classical) non-observable quantity (the vector potential) becomes observable (part of it) through a quantum non-local effect. Its gravitational analog allows a (Newtonian) non-observable quantity (the additional constant to the Newtonian potential) to become observable in the relativistic theory through the angular deficit in strings.

Considering $c=0$ and $n=1(\sigma=0)$ in (2.1) we have from (3.2-6) and (3.8)

$$
d s^{2}=-d \tau^{2}-2 b \sqrt{a} d \tau d \varphi+d r^{2}+d z^{2}+\left(\frac{r^{2}}{a}-b^{2} a\right) d \varphi^{2},
$$

producing a locally flat spacetime. In this case (7.7) represents the exterior spacetime of a spinning string along the axis of symmetry [27] with the same linear energy density $\lambda$ given by (7.5) and angular momentum $J$ given by

$$
J=-\frac{1}{4} b \sqrt{a}
$$


being $a>1$.

As it has been recently shown [27], a quantum scalar particle moving around a spinning cosmic string as given by (7.7), exhibits a phase factor proportional to $J$, in its angular momentum. An evident reminiscence of the Aharanov-Bohm effect. It is also worth mentioning that even if $b=0$, an Aharanov-Bohm like effect (of a different kind) appears (as commented in the static case), since the angular momentum spectrum differs from the usual one, if only $a>1$.

\section{Conclusion}

We obtained physical interpretations for the four real parameters $n, a, b, c$ appearing in the Lewis metric (3.2-5) for the Weyl class.

The parameter $n$ is associated to the Newtonian mass per unit length of an uniform line mass when it produces low densities.

The parameter $a$ is connected to the constant arbitrary potential that exists in the corresponding Newtonian solution. In the static and locally flat limit of the Weyl class when $a>1$ it produces a linear energy density along a string, being linked to the gravitational analog of the Ahanarov-Bohm effect. This effect demonstrates that gravitation depends on the topological structure of spacetime.

The parameter $b$, as we showed, is associated, in the locally flat limit, with the angular momentum of a spinning string generating one kind of the gravitational Aharanov-Bohm effect. 
The parameter $c$ is produced by the vorticity of the source of the Weyl class metric when it is represented by a general stationary completely anisotropic fluid. This parameter, together with the parameter $b$, is responsible for the non-staticity of the Weyl class metric.

We proved too, using the Cartan scalars, that only $n$ helps to curve spacetime locally and that the three parameters $a, b, c$ only influence the topological structure of spacetime. As a consequence, the stationary Lewis metric for the Weyl class is indistinguishable locally from the static Levi-Civita metric as far as the curvature of spacetime is concerned.

\section{Appendix A}

The non zero components of $R_{\mu \nu}$ for the metric (2.1) in the Van Stockum's notation [3] are

$$
\begin{aligned}
2 e^{\mu} D R_{0}^{0} & =\left(\frac{l f^{\prime}+k k^{\prime}}{D}\right)^{\prime}, \\
2 e^{\mu} D R_{0}^{3} & =\left(\frac{f k^{\prime}-k f^{\prime}}{D}\right)^{\prime}, \\
2 e^{\mu} D R_{3}^{0} & =\left(\frac{k l^{\prime}-l k^{\prime}}{D}\right)^{\prime}, \\
2 e^{\mu} D R_{3}^{3} & =\left(\frac{f l^{\prime}+k k^{\prime}}{D}\right)^{\prime}, \\
2 R_{11}=-\mu^{\prime \prime}+\mu^{\prime} & \frac{D^{\prime}}{D}-2 \frac{D^{\prime \prime}}{D}+\frac{k^{\prime 2}+f^{\prime} l^{\prime}}{D^{2}}, \\
2 R_{22} & =-\mu^{\prime \prime}-\mu^{\prime} \frac{D^{\prime}}{D},
\end{aligned}
$$

where the primes stand for differentiation with respect to $r$ and

$$
D^{2}=k^{2}+f l .
$$

The four equations $(A .1-4)$ are not all independent, any one of them can be expressed in terms of remaining three. 


\section{Appendix B}

We give below the components of the Riemann tensor in the standard Lorentz frame (5.4),

$$
\begin{gathered}
-R_{0101}=R_{2323}=\frac{1}{4}\left(n^{2}-1\right) r^{-\frac{1}{2}\left(n^{2}+3\right)}, \\
-R_{1313}=R_{0202}=\frac{1}{8}(n+1)\left(n^{2}-1\right) r^{-\frac{1}{2}\left(n^{2}+3\right)}, \\
-R_{0303}=R_{1212}=\frac{1}{8}(n-1)\left(n^{2}-1\right) r^{-\frac{1}{2}\left(n^{2}+3\right)} .
\end{gathered}
$$

\section{Appendix C}

The algebraic invariants of the Riemann tensor for vacuum spacetimes are [28] $R_{\alpha \beta \gamma \delta} R^{\alpha \beta \gamma \delta}$, $R^{* \alpha \beta}{ }_{\gamma \delta} R_{\alpha \beta}^{\gamma \delta}, R_{\alpha \beta \gamma \delta} R^{\gamma \delta \mu \nu} R_{\mu \nu}^{\alpha \beta}, R_{\gamma \delta}^{* \alpha \beta} R^{\gamma \delta \mu \nu} R_{\mu \nu \alpha \beta}$, where

$$
R_{\gamma \delta}^{* \alpha \beta}=\frac{1}{2} \frac{\epsilon^{\alpha \beta \mu \nu}}{\sqrt{-g}} R_{\mu \nu \gamma \delta}
$$

For the Weyl class metric the non zero algebraic invariants using (B.1-3) are

$$
\begin{gathered}
R_{\alpha \beta \gamma \delta} R^{\alpha \beta \gamma \delta}=\frac{1}{4}\left(n^{2}+3\right)\left(n^{2}-1\right)^{2} r^{-\left(n^{2}+3\right)}, \\
R_{\alpha \beta \gamma \delta} R^{\gamma \delta \mu \nu} R_{\mu \nu}^{\alpha \beta}=\frac{3}{16}\left(n^{2}-1\right)^{4} r^{-\frac{3}{2}\left(n^{2}+3\right)} .
\end{gathered}
$$

\section{Acknowledgment}

The authors acknowledge to Bill Bonnor and Kayll Lake for reading the manuscript and for usefull comments. MFAS and FMP gratefully acknowledge financial assistance from CAPES and $\mathrm{CNPq}$, respectively. 


\section{References}

[1] Lewis T 1932 Proc. Roy. Soc. Lond. 136176

[2] Kramer D, Stephani H, MacCallum M A H and Herlt E 1980 Exacty Solutions of Einstein's Field Equations (Cambridge: Cambridge University Press) p 221

[3] Levi-Civita T 1917 Rend. Acc. Lincei 26307

[4] Cartan E 1951 Leçons sur la Géométrie des Espaces de Riemann (Paris: Gauthier-Villars)

[5] Karlhede A 1980 Gen. Rel. Grav. 12693

[6] Bonnor W B and Martins M A P 1991 Class. Quantum Grav. 8727

[7]Bonnor, W B 1980 J. Phys. A: Math. Gen. 132121

[8] Stachel, J 1982 Phys. Rev. D 261281

[9] Lemos, J P S 1994 to be published

[10] Krasiński, A 1974 Acta Phys. Polon. B5 411

[11] Krasiński, A 1975 J. Math. Phys. 16125

[12] Krasiński, A 1978 Rep. Math. Phys. 14225 
[13] Krasiński, A 1975 Acta Phys. Polon. B6 223

[14] van Stockum, W J 1937 Proc. R. Soc. Edin. 57135

[15] MacCallum M A H and Skea J E F 1994 SHEEP: a computer algebra system for general relativity Algebraic Computing in General Relativity: Lecture Notes from the First Brazilian School on Computer Algebra vol 2 ed M J Rebouças and W L Roque (Oxford: Oxford University Press)

[16] MacCallum M A H 1991 Computer-aided classification of exact solutions in general relativity in General Relativity and Gravitational Physics (9th Italian Conference) ed R Cianci, R de Ritis, M Francaviglia, G Marmo, C Rubano and P Scudellaro (Singapore: World Scientific) p 318

[17] Paiva F M, Rebouças M J and MacCallum M A H 1993 Class. Quantum Grav. 101165

[18] Paiva F M 1993 PhD Thesis Centro Brasileiro de Pesquisas Físicas, Rio de Janeiro

[19] Frick I 1977 SHEEP users guide Report 77-11 Institute of Theoretical Physics, University of Stockholm

[20] Åman J E 1987 Manual for CLASSI-classification programs for geometries in general relativity (3rd provisional edn) University of Stockholm Report

[21] MacCallum M A H and Åman J E 1986 Class. Quantum Grav. 31133 
[22] Darmois E 1927 Mémorial des Sciences Mathématiques (Paris: Gauthier-Villars) Fasc 25

[23] Bonnor W B 1992 Gen. Rel. Grav. 24551

[24] Bonnor W B and Davidson W 1992 Class. Quantum Grav. 92065

[25] Linet B 1985 Gen. Rel. Grav. 171109

[26] Dowker J S 1967 Nuovo Cimento B 52129

[27] Jensen B and Kučera J 1993 J. Math. Phys. 344975

[28] Campbell S J and Wainwright J 1977 Gen. Rel. Grav. 8987 